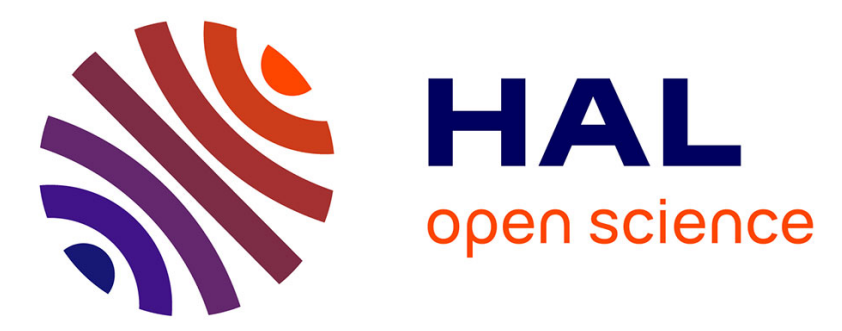

\title{
Coherent beam superposition of ten diode lasers with a Dammann grating
}

David Pabœuf, Florian Emaury, Sébastien de Rossi, Raymond Mercier, Gaëlle Lucas-Leclin, Patrick Georges

\section{- To cite this version:}

David Pabœuf, Florian Emaury, Sébastien de Rossi, Raymond Mercier, Gaëlle Lucas-Leclin, et al.. Coherent beam superposition of ten diode lasers with a Dammann grating. Optics Letters, 2010, 35 (10), pp.1515-1517. hal-00533598

\section{HAL Id: hal-00533598 https://hal.science/hal-00533598}

Submitted on 30 Mar 2012

HAL is a multi-disciplinary open access archive for the deposit and dissemination of scientific research documents, whether they are published or not. The documents may come from teaching and research institutions in France or abroad, or from public or private research centers.
L'archive ouverte pluridisciplinaire HAL, est destinée au dépôt et à la diffusion de documents scientifiques de niveau recherche, publiés ou non, émanant des établissements d'enseignement et de recherche français ou étrangers, des laboratoires publics ou privés. 


\title{
Coherent beam superposition of ten diode lasers with a Dammann grating
}

\author{
David Pabœuf, Florian Emaury, Sébastien de Rossi, Raymond Mercier, \\ Gaëlle Lucas-Leclin,* and Patrick Georges \\ Laboratoire Charles Fabry de I'Institut d'Optique, CNRS, Université Paris-Sud, \\ Campus Polytechnique-RD128-91127 Palaiseau Cedex, France \\ *Corresponding author: gaelle.lucas-leclin@institutoptique.fr
}

Received December 8, 2009; revised March 23, 2010; accepted March 30, 2010; posted April 6, 2010 (Doc. ID 121175); published May 5, 2010

\begin{abstract}
We demonstrate the use of a binary diffractive optical element in a very simple setup to convert the multilobed beam from a low fill factor array of coherent laser diodes into a quasi-Gaussian beam. The phase profile of the grating is determined with a phase retrieval algorithm. Experimentally, the conversion efficiency reaches more than $44 \%$. We also establish that this setup can be used to make an effective measurement of the coherency of the laser array. (C) 2010 Optical Society of America

OCIS codes: $140.2010,140.3298,050.1970$.
\end{abstract}

The maximum output power from a single laser, whether based on a fiber, bulk, or semiconductor amplifier, is limited by effects such as nonlinearities, thermal lensing, and optical material degradation. Using several lasers in parallel operating below the degradation limit appears as the most efficient solution to increase the output power at a reduced cost. Combination techniques as coherent beam combining are then necessary to scale the brightness [1]. Moreover, because of the nonuniform aperture in the near field, the far-field profile resulting from the coherent combination of several lasers is composed of interference peaks in which the total output power is distributed; its center intensity is proportional to the fill factor of the laser arrangement, defined as the ratio of the emitting area of one laser to that of the laser array. Since, for many applications, maximal on-axis far-field intensity is required, an efficient conversion of the multilobed beam into a Gaussian profile is necessary to further improve the beam quality. Diode laser arrays are particularly sensitive to this issue, as low fill factors are necessary to limit the thermal load and reach high output powers.

Several techniques aiming at converting the emission from a low-fill-factor coherent laser array into a single lobe have been investigated [2]. In this work we describe an entirely passive solution consisting in the superposition of laser beams by use of a diffractive optical element (DOE). Indeed, a proper design of the phase profile of the DOE provides a fine control of the amplitude and phase of these diffraction orders. Thanks to the reciprocity of light propagation, this optical device can also combine many coherent laser beams on top of one another as long as their phases, amplitudes, and angles of incidence match its diffraction pattern. The DOE may be used inside an external cavity to phase lock and combine the lasers [3] or outside the laser cavity to superpose the coherent laser beams $[4,5]$. The major drawback of the first configuration lies in the inherent additional intracavity losses due to the $<100 \%$ DOE transmission, which may become detrimental to the phase-locked operation of the laser array by reducing the actual coupling between emitters. In this Letter, we propose to coherently superpose the beams from an array of ten phased-locked diode lasers with a DOE outside the laser cavity. We also demonstrate that this technique provides an effective measurement of the coherent power within the overall laser emission.

The laser array consists of ten narrow indexguided tapered diode lasers characterized by a single emitter total width of $2 w=30 \mu \mathrm{m}$ at $1 / e^{2}$ and a pitch $p=100 \mu \mathrm{m}$. The phase-locked operation of the laser array is obtained through an extended cavity configuration, which induces a diffractive coupling between the emitters. The external cavity setup, which consists of a Talbot cavity closed by a volume Bragg grating, has already been described and fully characterized in [6]. Depending on the tilt of the output coupler, either in-phase or out-of-phase supermode operation can be obtained. A maximum output power of $1.7 \mathrm{~W}$ has been obtained at $4 \mathrm{~A}$ with in-phase mode operation. Because of the low fill factor (30\%) and the slightly multimode emission of the emitters, the far-field profile is composed of 15 interference peaks included in a $150 \mathrm{mrad}$ large envelope. The central lobe has a total width of $2.5 \mathrm{mrad}$ and contains $15 \%$ of the total power. The fringe visibility of the far-field pattern, defined as $V=\left(I_{\max }-I_{\min }\right) /\left(I_{\max }\right.$ $\left.+I_{\min }\right)$, where $I_{\max }\left(I_{\min }\right)$ is the maximum (minimum) intensity of the central lobe, is 0.9 close to the threshold and goes down to 0.8 at $4 \mathrm{~A}$. This confirms the good phase locking of all the emitters.

Our coherent combination setup is depicted in Fig. 1. The emission area of the phase-locked laser array is imaged onto the plane NF by a cylindrical afocal lens system working in the slow axis $\left(\mathrm{L}_{1}, \mathrm{~L}_{2}\right)$. An additional phase mask onto the $\mathrm{NF}$ plane is used to adapt the phases of each beam to match the diffraction pattern of the DOE. The DOE, placed on the Fourier plane of collimating lens $\mathrm{L}_{3}$, converts these ten beams into a single one. In the focal plane $\mathrm{NF}^{\prime}$ of $\mathrm{L}_{4}$, the imaged near-field profile consists of one main 

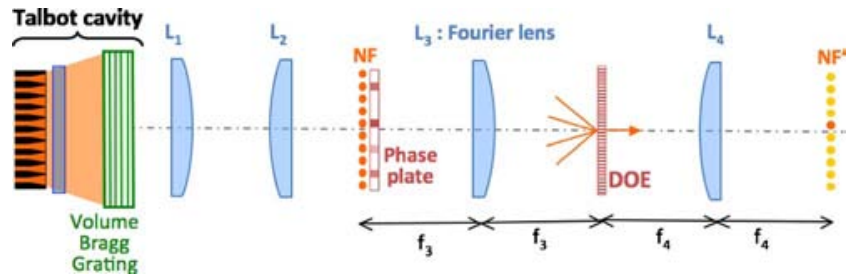

Fig. 1. (Color online) Experimental setup. $\mathrm{L}_{1}-\mathrm{L}_{4}$ are cylindrical lenses with focal lengths $f_{1}=f_{2}=100 \mathrm{~mm}, f_{3}$ $=50 \mathrm{~mm}, f_{4}=250 \mathrm{~mm}$.

peak containing most of the optical power. As a DOE, a simple binary $(\pi, 0)$ phase grating (or Dammann grating [7]) has been chosen. The amplitudes $\left(\tau_{n}\right)$ of the diffraction orders of such a grating can be expressed as [8]

$$
\begin{aligned}
& \tau_{0}=1-2 \sum_{s=1}^{N}(-1)^{s} \frac{x_{s}}{a}, \\
& \tau_{n}=\frac{1}{i \pi n} \sum_{s=1}^{N}(-1)^{s} e^{-2 i \pi\left(x_{s} / a\right)}, \quad n \in Z^{*},
\end{aligned}
$$

where $a=f_{3} \times \lambda / p$ is the period of the grating related to the focal lens of the Fourier lens $\mathrm{L}_{3}$ and $\left\{x_{s}\right\}$ are the $N$ phase shifting positions defining one pattern of the grating. The phase profile $\left\{x_{s}\right\}$ of this grating has been numerically determined so that its diffraction pattern matches the theoretical sinusoidal envelope of the near-field intensity profile of either the inphase or the out-of-phase mode following the classical expression [9]: $\cos ^{2}(\pi x /(M+1) p)$, where $M$ is the number of emitters. The phase state required in the near-field plane NF is given by the relative phases of the diffraction orders $\left\{\tau_{n}\right\}$ of the Dammann grating. The efficiency of the solution is evaluated by the combination efficiency $\eta$, defined as the ratio between the power combined into the main spot in $\mathrm{NF}^{\prime}$ (see Fig. 1) and the total optical power in that plane $\eta=P_{\text {comb }} / P_{\text {tot }}$.

Our best design, which consists in a pattern with only one $\pi$ phase step, $\left\{x_{1} / a=0.41, x_{2} / a=0.59\right\}$, results in a theoretical combining efficiency $\eta_{0}=83 \%$, similar to the one obtained in [5]. It requires a $\{\pi, 0, \pi, 0,0,0, \pi, 0, \pi, 0\}$ phase state in the NF plane, which corresponds to neither the in-phase mode nor the out-of-phase mode but is actually close to the latter with the fifth emitter phase shifted by $\pi$. A simple phase mask, with one $\pi$ phase step, has to be used in the NF plane (see Fig. 1) to adapt the phase state of the near-field profile. In Fig. 2, we compare the theoretical near-field profiles obtained in $\mathrm{NF}^{\prime}$ with and without the DOE for a mode with the previously determined phase state and a cosine intensity profile. Obviously, the DOE permits most of the optical power to be combined into a single spot. The remaining power is distributed into parasitic peaks corresponding to replicas of the diffraction orders of the grating.

The Dammann grating was manufactured by Holoeye Photonics according to our design. From its diffraction pattern we deduce a diffraction efficiency

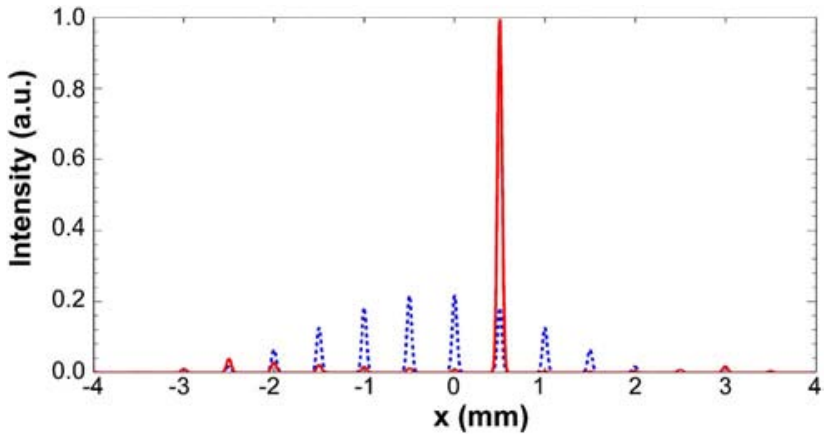

Fig. 2. (Color online) Theoretical near-field profiles at the output of the conversion setup in $\mathrm{NF}^{\prime}$. Blue dashed curve, without the DOE; red solid curve, with the DOE.

$\eta_{0}=83 \%$ in accordance with the theoretical value. The phase mask was realized in our laboratory by ion etching of a BK7 plate. It consists of a phase step of $0.99 \pi$ at $\lambda=976 \mathrm{~nm}$, and a width of $80 \mu \mathrm{m}$. Neither the phase plate nor the Dammann grating are antireflection coated; we evaluate the transmission of the optical system to $T=85 \%$. The overall combining efficiency is then given by $\eta=C \eta_{0}$, where $C$ is the effective power ratio contained in the array supermode. We first set the laser array close to threshold: $I=1.6 \mathrm{~A}, \quad P_{\text {laser }}=340 \mathrm{~mW}$ at the output of the extended-cavity laser, the volume Bragg grating being aligned to force out-of-phase mode operation. The near-field profile of the phase-locked array in the plane $\mathrm{NF}^{\prime}$ obtained with the DOE is compared with the pattern obtained without it in Fig. 3. As expected, with the DOE it exhibits a strong peak with low sidelobes; the experimental combining efficiency is $\eta$ $=44 \%$. This lower efficiency is attributed to imperfect phase locking $(C<1)$ of the lasers in the Talbot cavity and is detailed in the next paragraph. At higher operating currents, owing to stronger competition with self-running operation and the slightly multimode emission of the lasers, $\eta$ decreases but still remains higher than $35 \%$. At the maximum operating current $\left(I=2.9 \mathrm{~A}, P_{\text {laser }}=984 \mathrm{~mW}\right)$ possible at the time of the experiment because of a degradation of the diode laser bar, the power contained in the major peak is evaluated to $P_{\text {comb }}=365 \mathrm{~mW}$ after correction for the transmission of the optical system. The beam quality factor of the combined beam has been measured to

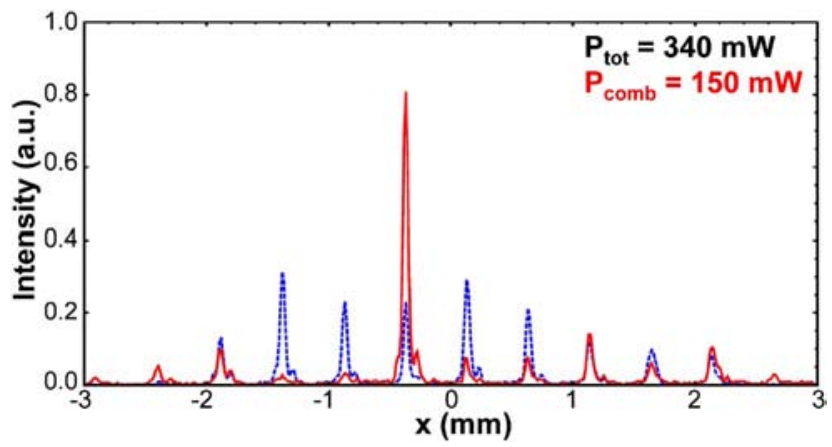

Fig. 3. (Color online) Experimental near-field profiles at the output of the system. Blue dashed curve, without the DOE; red solid curve, with the DOE, at operating current $I=1.6 \mathrm{~A}$ 
$M^{2}=1.7$ in the slow axis, with a slit selecting the peak of interest.

It is noteworthy that the combination setup provides a direct measurement of the coherent compound $C$ through the measurement of the overall combining efficiency $\eta$. The experimental evaluation of $C$ has been compared with theoretical evolution, as deduced from the visibility $V$ of the far-field profile, which is a standard criterion to estimate the coherence. The far-field profile of a phase-locked array of lasers may be described as the superposition of an incoherent, Gaussian shaped, intensity profile $I_{\text {inc }}(\theta)$ related to the self-running of the lasers and a coherent supermode far-field profile $I_{\mathrm{coh}}(\theta)$ [9]:

$$
\begin{gathered}
I_{\mathrm{inc}}(\theta)=\exp \left(\frac{-2 \pi^{2} w^{2} \sin ^{2}(\theta)}{\lambda^{2}}\right), \\
I_{\mathrm{coh}}(\theta)=I_{\mathrm{inc}}(\theta) \times\left(\frac{\sin \left(\frac{M}{2}\left(\frac{2 \pi p}{\lambda} \sin (\theta)+\frac{k \pi}{M+1}\right)\right)}{\sin \left(\frac{1}{2}\left(\frac{2 \pi p}{\lambda} \sin (\theta)+\frac{k \pi}{M+1}\right)\right)}\right. \\
\left.+(-1)^{k} \frac{\sin \left(\frac{M}{2}\left(\frac{2 \pi p}{\lambda} \sin (\theta)-\frac{k \pi}{M+1}\right)\right)}{\sin \left(\frac{1}{2}\left(\frac{2 \pi p}{\lambda} \sin (\theta)-\frac{k \pi}{M+1}\right)\right)}\right)^{2}
\end{gathered}
$$

with $k=1$ for the in-phase mode and $k=M=10$ for the out-of-phase mode. $C$ is thus the ratio of the coherent power to the total power of the laser array. The visibility has been computed from these far-field profiles for different values of $C$ (see Fig. 4), in the out-ofphase mode. A visibility $V$ of 0.8 corresponds to $50 \%$ of the coherent power, which leads to a combining ef-

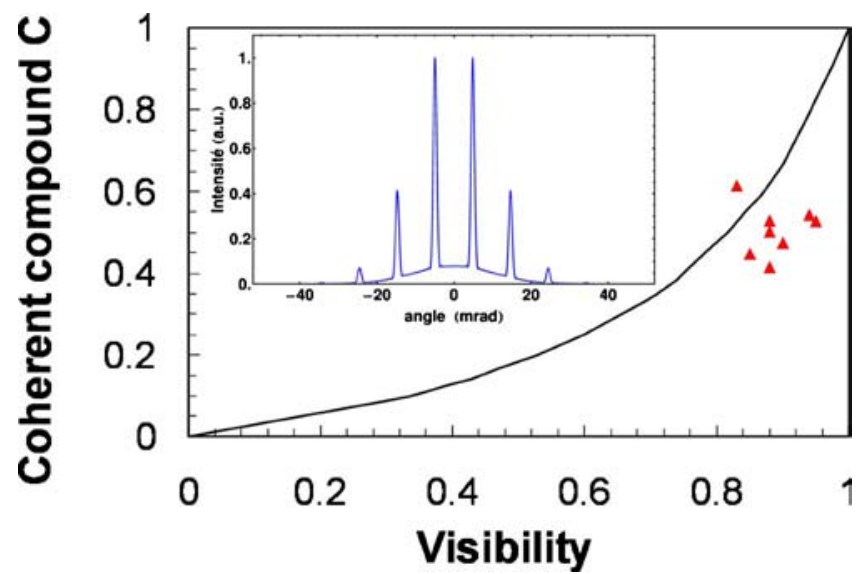

Fig. 4. (Color online) Evolution of the coherent compound $C$ with visibility $V$ (black curve, theoretical evolution; triangles, experimental measurement). Inset, simulated farfield profile of the out-of-phase mode with $C=0.4$. ficiency $\eta=C \eta_{0}=41 \%$, in agreement with our experimental results.

Experimentally, the visibility $V$ of the far-field profile at the output of the Talbot external cavity has been determined for different operating currents; in parallel, the combining efficiency $\eta$ in the $\mathrm{NF}^{\prime}$ plane has been measured, from which we deduce the experimental coherence $C$. Figure 4 compares the theoretical and experimental evolution of $C$ with $V$. The measured proportion of coherent power $C$ remains close to $50 \%$ whatever the fringe visibility. We attribute the difference between the experimental and the theoretical evolutions to the contribution of other supermodes that do not affect the fringe visibility but that cannot be converted in a single beam by our setup, since their phase state does not match the diffraction pattern of the DOE. Moreover, the emitters being slightly multimode, the experimental far-field pattern is larger than the one that was considered for the design of the grating.

In summary, we have demonstrated the use of a DOE to combine the beams from an array of ten coherent tapered diode lasers. A simple setup with a Dammann grating and a phase plate has been developed. A maximum output power of $365 \mathrm{~mW}$ in a quasi-Gaussian beam with an $M^{2}$ of 1.7 has been obtained. Our configuration, which sets apart the phase-locking of the emitters and their coherent superposition, appears fully adequate for high-power arrays. In addition this setup characterizes the emission of the phase-locked laser array by providing an effective measurement of the amount of power emitted in one supermode of the array, which leads to a more accurate evaluation of the phase-locking quality than the usual analysis of the far-field profile.

This work was supported within the European project WWW.BRIGHTER.EU under grant FP6-IST035266. D. Pabœuf thanks the French Ministry of Defense (DGA) for funding his Ph.D. The authors are thankful to A. Jérôme for his technical support.

\section{References}

1. T. Y. Fan, IEEE J. Sel. Top. Quantum Electron. 11, 567 (2005).

2. M. Khajavikhan, A. Hoyer-Leitzel, and J. R. Leger, Opt. Lett. 33, 2377 (2008).

3. J. R. Leger, G. J. Swanson, and W. B. Veldkamp, Appl. Opt. 26, 4391 (1987).

4. E. C. Cheung, J. G. Ho, G. D. Goodno, R. R. Rice, J. Rothenberg, P. Thielen, M. Weber, and M. Wickham, Opt. Lett. 33, 354 (2008).

5. G. Hergenhan, B. Lucke, and U. Brauch, Appl. Opt. 42, 1667 (2003).

6. D. Paboeuf, G. Lucas-Leclin, P. Georges, N. Michel, M. Krakowski, J. Lim, S. Sujecki, and E. Larkins, Appl. Phys. Lett. 93, 211102 (2008).

7. H. Dammann and K. Görtler, Opt. Commun. 3, 312 (1971)

8. S. Martellucci and A. N. Chester, eds., Diffractive Optics and Optical Microsystems (Plenum, 1997), p. 422.

9. J. K. Butler, D. E. Ackley, and D. Botez, Appl. Phys. Lett. 44, 293 (1984). 